\title{
Arthroscopic approach and anatomy of the hip
}

\author{
Alessandro Aprato \\ Matteo Giachino \\ Alessandro Masse
}

School of Medicine, University of Turin, Turin, Italy

Corresponding author:

Alessandro Aprato

Viale 25 Aprile 137

10131 Torino, Italy

E-mail: ale_aprato@hotmail.com

\section{Summary}

Background: Hip arthroscopy has gained popularity among the orthopedic community and a precise assessment of indications, techniques and results is constantly brought on.

Methods: In this chapter the principal standard entry portals for central and peripheral compartment are discussed. The description starts from the superficial landmarks for portals placement and continues with the deep layers. For each entry point an illustration of the main structures encountered is provided and the principal structures at risk for different portals are accurately examined. Articular anatomical description is carried out from the arthroscope point of view and sub-divided into central and peripheral compartment. The two compartments are systematically analyzed and the accessible articular areas for each portal explained. Moreover, some anatomical variations that can be found in the normal hip are reported.

Conclusion: The anatomical knowledge of the hip joint along with a precise notion of the structures encountered with the arthroscope is an essential requirement for a secure and successful surgery. Level of evidence: $\mathrm{V}$.

KEY WORDS: hip arthroscopy, anatomy, hip portals.

\section{Introduction}

Hip arthroscopy has to cope with characteristic technical issues due joint anatomy; articular depth, narrow maneuvering space and the proximity of hazardous structures have led in the last decades to a constant re- finement of surgical accesses and instrumentation. The comprehension of bone anatomy and soft tissues surrounding the hip is the fundamental pre-requisite for performing a safe and effective hip arthroscopy. The individuation of the correct entry-points or portals represents the first step for an optimal approach to the hip. More than 18 portals have been historically reported according to literature, but most of the Authors believe that only 9 of them have an essential role, since part of the remnants are not sufficiently described, tested for safety, or reproducible ${ }^{1}$. Safety and reproducibility were, in fact, the principles for the detection of the nine principal portals used today as reference for hip arthroscopy. The awareness of the soft tissues and delicate structures involved before entering hip compartments derives from several anatomical studies and cadaveric dissections performed by Authors throughout the years.

\section{Portals}

As the patient is positioned in traction on the surgical table with neutral rotation of the affected limb, the principal superficial landmarks of the hip are identified. Laterally the greater trochanter (GT) is easily felt and its anterior and posterior edges outlined until they merge superiorly in the tip. Proximally the iliac crest has to be palpated and the anterior superior iliac spine (ASIS) identified. Both bony landmarks are generally recognized without difficulty even in obese patients as they are particularly superficial. A line may be drawn from the ASIS down to the center of the patella, representing the medial border for safe accesses; beyond this line there is a higher risk of femoral nerve and femoral vessels injuries ${ }^{2,3}$.

The first portal to be marked is generally the anterolateral $(\mathrm{AL})$ one, which is placed $1 \mathrm{~cm}$ proximal and 1 $\mathrm{cm}$ anterior to the tip of the GT. The postero-lateral $(\mathrm{PL})$ portal is located in line with the AL mirrored posteriorly to the tip of the GT. The third main portal, the anterior portal (AP), is located at the intersection of a transverse line passing through the $\mathrm{AL}$ and $\mathrm{PL}$ portals and a sagittal line drawn distally from the ASIS. These are the three standard entry points for central compartment, historically described by Byrd and generally taken as reference for obtaining the others ${ }^{4}$. On the distal vertex of the equilateral triangle constructed on the AL-AP segment lies the mid-anterior portal (MAP), while the reflected proximal point correspond to the proximal mid-anterior portal (PMAP) (Figure 1) ${ }^{1}$. 


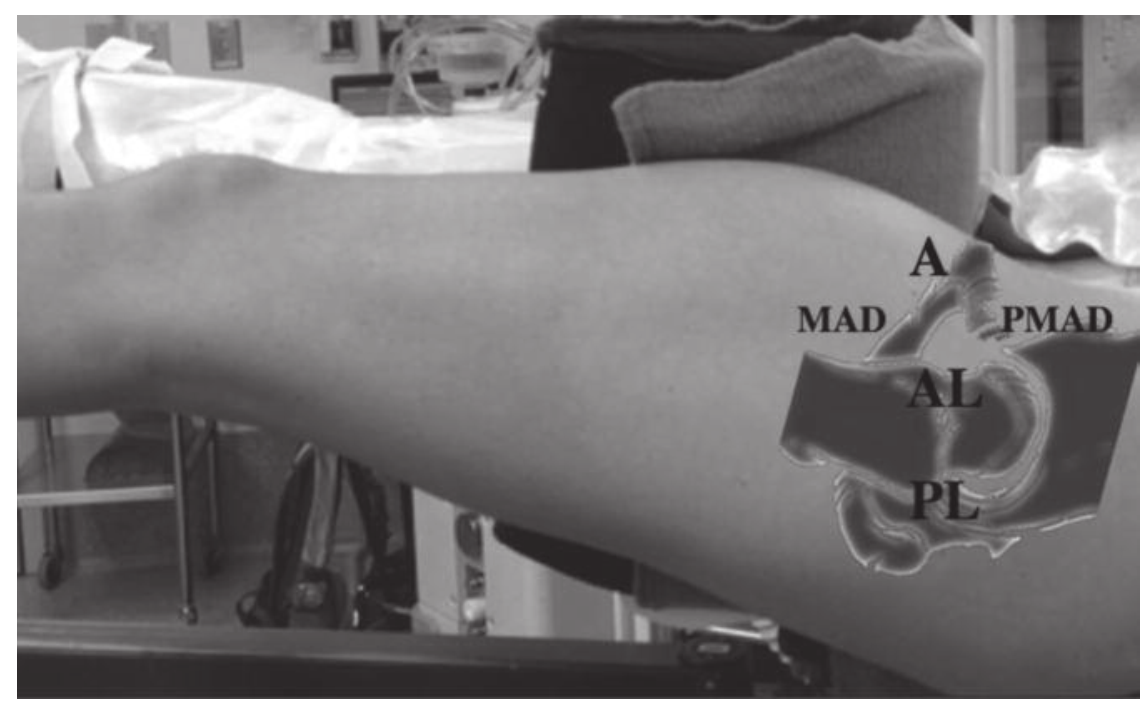

Figure 1. Principal portals.

The space confined to the joint itself is historically defined as the central compartment, whose main structures are articular cartilage, ligamentum teres, transverse ligament and inner acetabular rim. Laterally to the central compartment there is the peripheral compartment. It is still located into articular capsule but it is distinguished from the central one for specific anatomical and pathological features. Femoral neck, capsule and the outer acetabular rim are the major structures of the peripheral compartment. In this way with 5 portals to the central and peripheral compartments are obtained. Different insertion angles of the instruments lead to the different compartments as shown in Table I.

AL portal crosses superficially the gluteal fascia at its junction with tensor fascia latae before deepening through the fibers of the gluteus medius muscle. If driven in the medial compartment, it pierces the antero-lateral side of the capsule. The AP enters in the muscle belly of sartorius and rectus femoris muscles, if placed in the traditional location; a more recent in- terpretation of AP is slightly more lateral (ca. $1 \mathrm{~cm}$ ) and crosses tensor fascia latae before intersecting the interval between gluteus minimus and rectus femoris. Deeply it goes into the anterior capsule, laterally to the anterior acetabular rim. MAP for both central and peripheral compartments travels across the same musculo-tendinous structures of AP, though the direction of the tunnel is clearly more lateral-tomedial, having the latter a significant impact on the visualization of the acetabulum. PL portal pierces the gluteal fascia and crosses the gluteus medius; medially to the GT, it passes antero-superiorly to the piriformis tendon before entering the lateral capsule in its posterior aspect ${ }^{1,4}$.

\section{Neurovascular structures}

Hip arthroscopy has the peculiarity of being performed at a bigger depth if compared to the other joints. The impossibility of a direct palpation of the

Table I. Portals approximate insertion angle for central and peripheral compartments ${ }^{1}$.

\begin{tabular}{ll}
\hline Portal & Approximate insertion angle \\
\hline Central Compartment & \\
AP & $35^{\circ}$ cephalad, $35^{\circ}$ posterior \\
AL & $15^{\circ}$ cephalad, $15^{\circ}$ posterior \\
MAP & $35^{\circ}$ cephalad, $25^{\circ}$ posterior \\
PL & $5^{\circ}$ cephalad, $5^{\circ}$ anterior \\
\hline Peripheral Compartment & \\
AL & $15^{\circ}$ cephalad, $5^{\circ}$ anterior \\
MAP & $15^{\circ}$ cephalad, $20^{\circ}$ posterior \\
PMAP & $40^{\circ}$ caudad, $25^{\circ}$ posterior \\
PL & $25^{\circ}$ caudad, $15^{\circ}$ anterior \\
\hline
\end{tabular}


joint makes instrument maneuvering and portals setting almost a blind operation. For this reason, the use of a C-arm is recommended. Even under X-rays control, there is still some concern on the potential neurovascular injuries that may happen in a region where threatening structures are so represented. Different studies over the last years tried to assess the relationships between portals and major structures at risk, here we summarize those findings to support the surgeon for practicing as safe as possible ${ }^{1-7}$.

The principal dangers come from deep and superficial vessels and nerves surrounding the hip. On the anterior side of the joint, medially to the acetabulum lie the neurovascular bundle; the most lateral element is the femoral nerve, associated with femoral artery and vein proceeding from lateral to medial. Remaining on the anterior aspect of the hip but more superficial, the lateral femorocutaneous nerve (LFCN) emerges from underneath the inguinal ligament just medial to the ASIS and courses down along the surface of the sartorius muscle (Figure 2).

The proximal path of the nerve is relatively constant but its branching is rather irregular, giving 0 to 5 branches when becoming more superficial; the most lateral of them are potentially on the course of AP. Peritrochanteric femoral arteries arise from medial and lateral circumflex arteries (MCFA, LCFA), branches of the profunda femori artery (Figure 3 ). Along the anterior face of the GT run the transverse and the ascending branches of the LCFA, possibly on the trajectory of anterior accesses. On the posterior side of the GT runs the critical ascending branch of the MCFA. The major nerve of the posterior space of the hip is certainly the sciatic nerve, leaving the pelvis accompanied by the piriformis muscle and then turning downward on the lateral rotators of the hip. A further nerve emerging from the ischiatic notch is the superior gluteal nerve, which supplies gluteus medius and minimus.

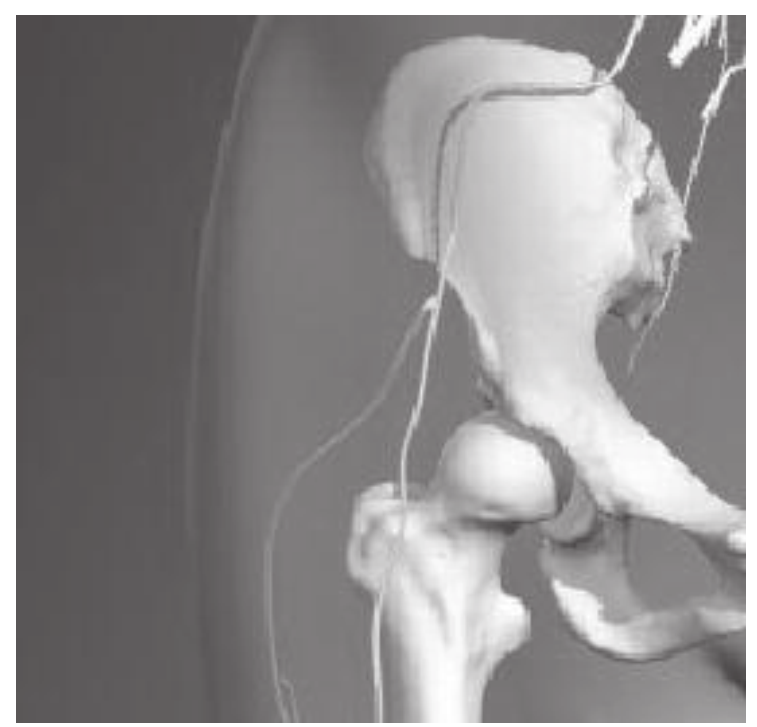

Figure 2. Lateral femorocutanous nerve.

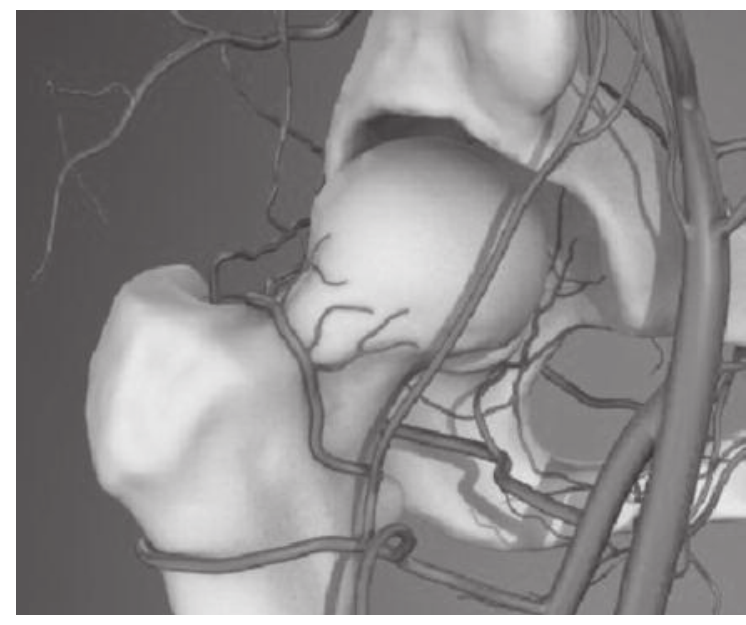

Figure 3. Vascular anatomy of the hip.

Relations of these structures with arthroscopic portals have to be assessed and topographic knowledge of the delicate anatomy surrounding the hip fully understood.

$A P$ : the LFCN is the principal structure at risk. Its high anatomical variability and unpredictable course involve a concrete possibility of being really close to a lateral branch of the nerve. Some Authors place the AP one $\mathrm{cm}$ lateral to the standard point in order to reduce this chance. Others suggest that avoiding excessively deep stab wounds and a respectful dissection when preparing the accesses protect the nerve from laceration. The main consequence of nerve damage is painful neuroma or neuroapraxia. The femoral nerve route is always at a safe constant distance from AP, even if at the level of the capsule they get closer to each other. The medial large femoral vessels are considered at a secure location. The ascending branch of the LCFA usually ends distally to AP course; however, it is described that a little terminal branch could extend proximally towards the portal, even crossing it. Despite that, no reports of major bleeding have been recorded due to this vessel.

$A L$ portal: the path followed by this access is generally in a safe area. The two important neurovascular structures nearby its course are the superior gluteal nerve and the LFCN. The first exits from the greater sciatic notch and runs transversely along the inner surface of the gluteus medius from posterior to anterior. For its entire route the superior gluteal nerve is at a safe distance from the portal. Lateral branches of the LCFA are far too medial to be a real threat. For its safety $A L$ portal is frequently chosen as first arthroscope entry point.

$M A P$ : the nervous structures encountered by MAP are essentially the lateral branches of the LCFN that can be close. Same carefulness should be employed as for the AP. The major vessel nearby the MAP is the terminal branch of the ascending LCFA, which is often relatively near. Authors report that a significant peri or post-operative bleeding due to its damage is 
unlikely to happen; furthermore these branches have a negligible role in femoral head blood supply and their interruption has no consequences.

$P L$ portal: the distance from the sciatic nerve decreases as instruments get closer to the capsula, however it remains reasonably safe. The delicate MCFA nears the portal around the piriformis tendon; it never gets too close to be at real risk and most important the postero-superior border of the GT seems to protect the artery from accidental wounding.

\section{Arthroscopic hip anatomy}

A proper description of the hip joint anatomy should be carried out from the arthroscopic perspective. Intrinsic arthroscopic limitations entail that only a limited field of view is achievable, although technical expedients are generally employed for enhancing every access. It is anyway paramount for the surgeon to be familiar with the anatomy of the portals and with their maneuvering limits. An excellent anatomical knowledge of the articular and periarticular region is the essence for practicing a safe surgery. Hip arthroscopy is nowadays considered a secure procedure if performed with modern techniques and proper instrumentations. Only in recent times standardized approaches for articular inspection have been proposed, since a rigorous identification of joint structures should lead to a better detection of pathological conditions $^{6}$. Along with the complete observation it is appropriate to palpate the structures to assess consistence and stability of tissues and to reveal pathological conditions.

Acetabular cavity description is commonly based on the clock face method, which takes as reference the center of the ligamentum transversus representing the 6 o'clock. A good technique for uniforming left and right hip is to ordinate counterclockwise the left hip, so that numbers from 1 to 5 correspond on both sides to the anterior acetabular rim. A further method is the description of geographical zone of acetabulum and femoral head, which allows a precise subdivision of articular surfaces ${ }^{8}$.

\section{Central compartment anatomy}

Exploration of the central compartment has been described in several papers ${ }^{1-12}$, hereby we describe the vision from the most commonly used portals:

$A L$ : this portal is often chosen as the first one for instruments because of its safety and the relatively easy effort for penetrating the joint. This can minimize the chance of iatrogenic damage to cartilage or labrum. From this point of view the deepest element that can be seen is the medial wall of the acetabulum; this area contains the shallow cotyloid fossa with ligmentum teres connecting its bottom to the fovea capitis (Figure 4). Its length is approximately $30-35 \mathrm{~mm}$ with a diameter of $10 \mathrm{~mm}$ and its higher tension is achieved with external rotation of the thigh. Synovial membrane envelopes the ligamentum. In the acetabular fossa the yellowish fibro-fatty tissue pad of pulvinar may also be visualized. If present, intra-articular free bodies are frequently found in this area. The portions of articular surface of femoral head and acetabulum that can be appreciated from this portal are located around the insertions of the ligamentum teres on both sides. The physiological hyaline cartilage is an uniform and smooth tissue that covers the surfaces of the joint, except for the cotyloid fossa and the insertions of ligamentum teres. Some findings that could interrupt cartilage uniformity are possible and should be known: firstly the traces of the triradiate cartilage fusion are occasionally observed over the acetabulum, they generally appear as grooves on articular surface and should be not confused as a

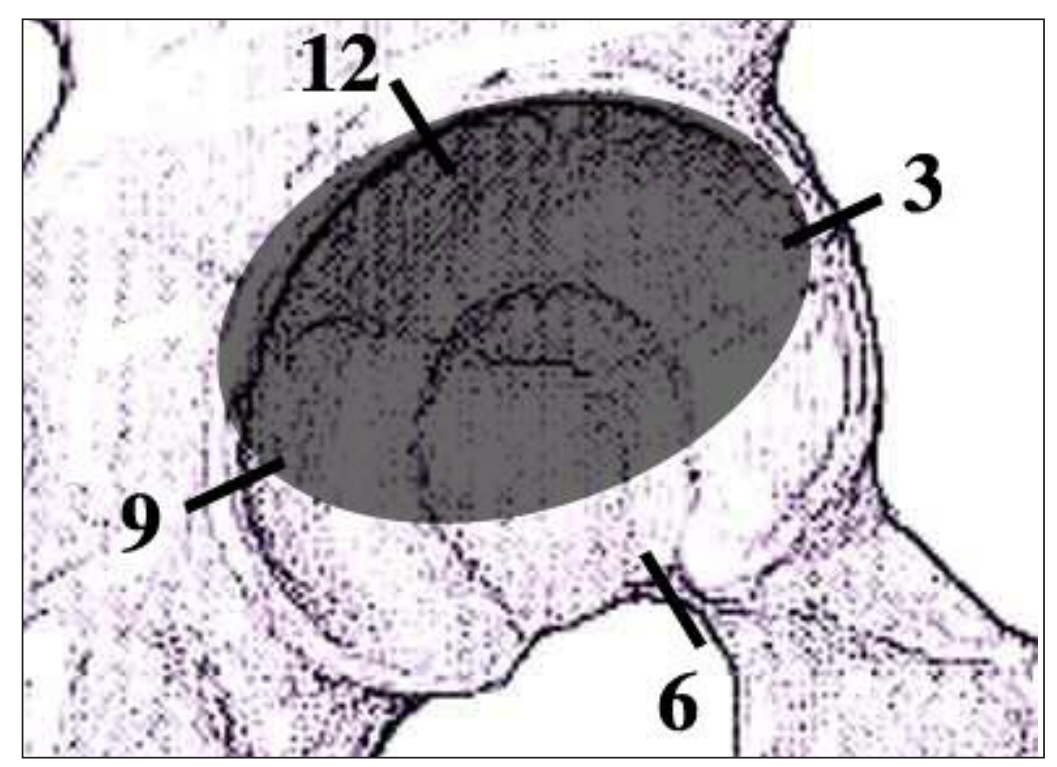

Figure 4. Anterolateral portal view. 
chondral lesion. Another anatomical variant of acetabular surface is the "stellate crease", an area about $1 \mathrm{~cm}$ wide positioned at 12:30-1 o'clock on acetabular roof. It's constituted of fibrous cartilage and again it should not be considered as pathological. Other known anatomical variations are the supra-acetabular fossa and the supra-acetabular notch, both variants don't induce any tissue reaction nor have pathological consequence.

From the previous position the arthroscope can be moved posteriorly to view the postero-medial acetabular surface and the posterior labrum. By moving the camera on the anterior aspect and rotating the lens superiorly the structure to be detected is the anterior triangle. The landmarks of this area are femoral head, the anterior capsule and the anterior labrum. The direct view of the anterior capsule at this level provides a secure camera-assisted detection of the optimal entry point for anterior portal.

At this point if the arthroscope is retracted the best view for the anterior labrum is offered. The acetabular labrum is a fibro-cartilaginous structure extended over the bony rim of acetabulum for almost its entire length. The labrum is actually interrupted inferiorly by the acetabular fossa and bridged by the transverse ligament. Acetabular labrum has a triangular shape on crosssection, its width is generally $5-8 \mathrm{~mm}$, with quite substantial variations in presence of a pathological hips. Additionally, the labrum is wider antero-superiorly and thicker in the postero-superior segment. Vascularization of acetabular labrum is provided by vessels coming from its bone insertion and from synovial membrane on the external border. The central arthroscopic view doesn't allow for direct visualization of labral blood supply because the inner face at the chondrolabral junction doesn't have visible vessels. The innervation of acetabular labrum is richly represented and is constituted by sensitive fibers and proprioceptive terminations. A crucial structure in arthroscopic hip examination is the chondrolabral junction, due to its implications in hip impingement pathology. The connection between labrum and chondral surface is tight at the posterior portion of the acetabular rim and it's frequently difficult to distinguish the two structures. On the contrary, when moving on the anterior and lateral portions, the labrum seems to have a looser anchoring to the chondral rim; labral tears and cartilage damages due to impingement are frequently found in this region. For best observing this anatomical characteristic it could be useful to inspect the chondrolabral junction with an arthroscopic hook probe, experiencing the boundary groove becoming harder to identify proceeding from anterior to posterior. When searching for labral tears another frequent anatomical variant should be considered, cases do in fact exist of a physiological sublabral sulcus (Figure 5), especially observed in the posterior quadrants. Size and depth of these breaches are variable, but the principal anatomical difference from pathological labral detachments is considered the absence of simultaneous chondral or labral lesions.

From AL portal it is then possible to visualize the external side of the labrum where it blends with fibers

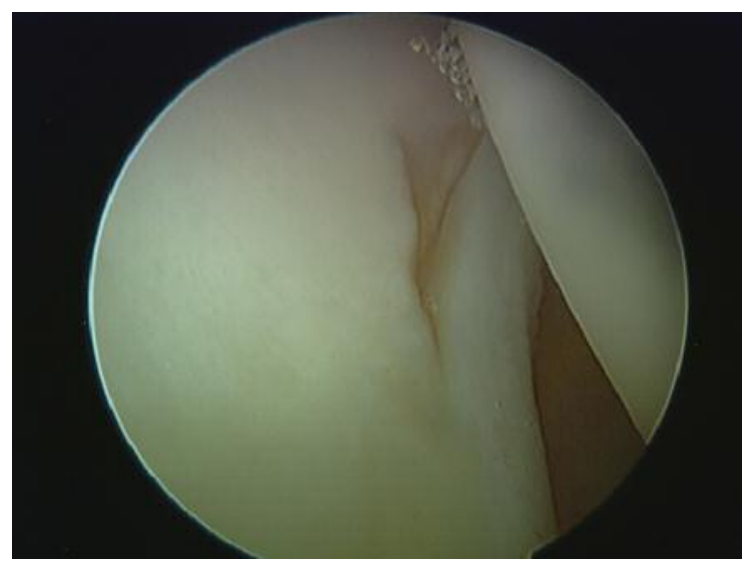

Figure 5. Posterior chondrolabral sulcus.

arising from the capsule insertion. The acetabular attachment of the capsule can be observed all around the accessible acetabular rim, extending the lateral view until the zona orbicularis posteriorly. If the camera is slightly withdrawn and focused on the femoral head, the round-shaped articular cartilage can be inspected. The head can be examined from its inferior to superior margin and the surface, as well as the acetabulum, should be smooth and even. The gap between femoral head and acetabular cavity can also be taken for monitoring the traction intensity applied to the inferior limb.

$A P$ : the anterior portal offers fine scenery of the acetabular fossa and the structures contained. The ligamentum teres can be fully appreciated from its insertion to its origin, uncovering even minor lesions if put in tension. The acetabular wall surface is also generally well inspected from the anterior portal. If enough traction is applied so that femoral head is sufficiently lateralized, the operator can inspect the transverse ligament of the acetabulum placed at 6 o'clock. It bridges the anterior and posterior end of the labrum with flattened and robust fibers covering the acetabular notch. Directing the lens laterally, it can be appraised the inner side of the AL portal; a further movement towards the posterior region reveals the optimal entry point for PL portal, located on the postero-lateral capsule. The direct visualization of the entry-point grants a safe placement of the access. The AP offers a proper view of all these structures, however the principal limitation to instruments maneuvrability is due to the anterior acetabular rim, which restricts the lateral-to-medial direction of the instruments. A wider working range with preservation of the field of view is generally given by MAP, that allows also for optimal anchors placement (Figure 6).

$P L$ portal: as the camera is inserted through this portal the first element coming into view is the posterior labrum. On the external side of the labrum it can be followed the shallow depression of the posterior capsule included between the capsular insertion and the zona orbicularis. Femoral head should be inspected from this perspective for completing the visible areas (Figure 7). The examination of the supero-lateral re- 

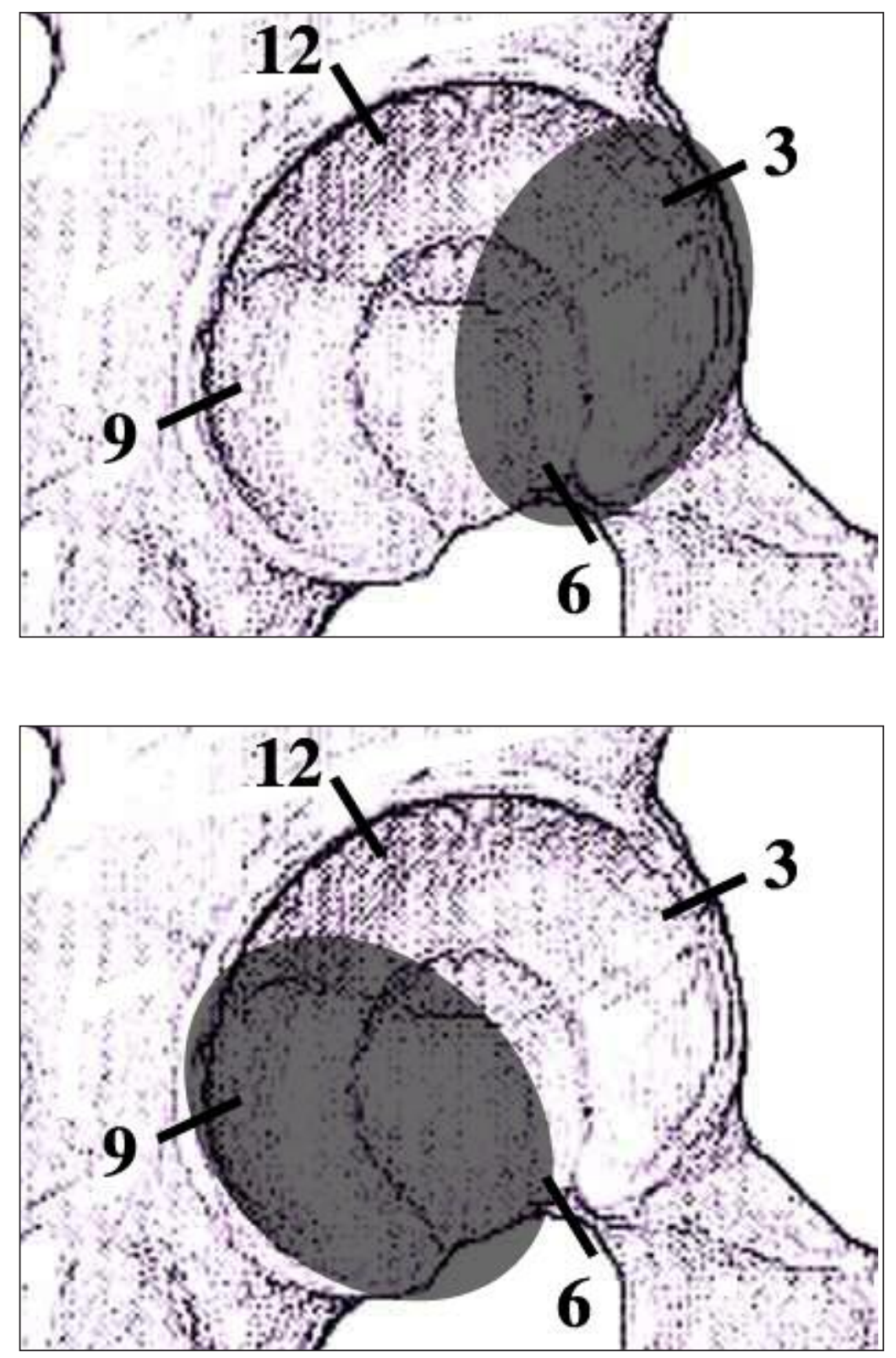

Figure 6. Anterior portal view.

Figure 7. Posterolateral portal view. gion of acetabular cartilage shall be performed, as it correspond to the weight-bearing zone, or dome, firstly affected by cartilage degeneration in arthritic alterations. This may be useful to evaluate minimal posterior wall fractures or traumatic labral tear (i.e. after a posterior dislocation).

\section{Peripheral compartment anatomy}

Anatomy of the peripheral compartment has been extensively described ${ }^{1-10,13-16}$. To easy the approach to peripheral compartment traction may be loosen, flexion of the hip further increases the maneuvering space, while the release of leg rotations allow for complete inspection for medial and lateral aspect of the compartment. The anatomical structure that most influences peripheral space is the joint capsule, the complete comprehension of its anatomical properties should guide the surgeon to minimize capsulotomy and the risk for post-operative instability. Hip capsule is a thick and tense fibrous sleeve extending from the lateral neck to the outer acetabular rim. The inner surface is entirely covered with synovial membrane, which can be involved in pathological processes. Some portions of the capsule have an increased strength as they are thicker. The supero-lateral part is reinforced by the fiber of the reflected tendon of rectus femoris. Other three longitudinal capsular reinforcements can be recognized: on the antero-lateral aspect of the capsule there is the iliofemoral ligament, on the antero-medial the pubofemoral ligament and on the posterior capsule the ischiofemoral liga- 
ment. These three ligaments are composed of fibers running parallel to the femoral neck axis; at neckhead junction the longitudinal fibers meet a further layer of circular fibers, this capsular reinforcement is the zona orbicularis and consists of a thicker stratum of annular fibers crossing the longitudinal ligaments and strengthening the retaining action of the capsule. The zona orbicularis has a major influence in sustaining hip stability, along with the other capsular thickenings; the knowledge of the key stabilizers of the capsule is essential when performing capsulotomy. An uncontrolled incision when practicing the arthroscopic accesses may lead from micro-instability until subluxation of the joint, especially if the zona orbicularis is interrupted.

Peripheral compartment can be approached from either the anterior portals or the PL. Since the principal pathologies of this area affect the anterior, lateral, or medial district of the femoral neck, the most used entry points are $\mathrm{AL}$ and MAP, which generally allow an ideal detection and treatment. If a posterior disorder has to be examined the PL portal shall be used. If observed from the anterior portals the peripheral compartment can be divided into three sections: medial, anterior and lateral.

The medial region is adequately explored when the hip is flexed and externally rotated, so that the femoral neck is moved forward. The first elements coming into view are generally the medial aspects of the neck, head and acetabular labrum, along with the antero-medial capsule, that shows a distinct zona orbicularis at this level. If the camera is pushed beyond the circular limit of the zona orbicularis, the space of the medial gutter can be visualized, as it is expression of the capsular recess nearby acetabular rim. The hunt for loose bodies should take into account this area, if their presence is suspected. The other significant structure of the medial region is the medial synovial fold, consisting of a plica of connective tissue spanning from the antero-medial sector of the head until the lesser trochanter. The edge of the medial neck, besides the zona orbicularis and the medial synovial fold are reliable landmarks when introducing the arthroscope for assessing its location into peripheral compartment.

The anterior region is explored raising the camera along the anterior border of the neck. From here it can be observed the antero-lateral neck continuing into the anterior head, which is enclosed by the labrum. At labral insertion it can be spotted the synovial membrane with its nutrient vessels responsible for main labrum vascularization disposed radially around it. In normal conditions the perfect congruence between labrum and femoral head create a perfect sealing mechanism that is evident when traction is released. In the antero-lateral region it's much more frequent to find disorders responsible for femoro-acetabular impingement and the indirect signs of its presence. An important maneuver while looking at the anterior region is performing dynamical tests of the joint, if an impingement is suspected or just corrected. The direct view of the conflicting parts is actually a precious support for both planning and checking the procedure.

The access to the lateral region of peripheral compartment is facilitated if the hip is internally rotated and flexion is slightly reduced. The lateral aspect of femoral neck should come into view as the camera is rotated posteriorly; this area is characterized by the presence of the lateral synovial folds. Like the medial one, those plicae run parallel to the neck along its longitudinal axis. The lateral folds are not a merely anatomical finding but an essential landmark for locating the retinacular vessels: those are, in fact, the terminal branches of the deep medial circumflex artery, the major responsible of femoral head vascularization. Arterial pulse within the fold can be generally spotted and vessels identified. Preservation of the retinacular blood supply during procedures involving the neck (especially the resection of femoral bump in cam impingement) is of primary importance to avoid avascular necrosis of femoral head.

\section{Conclusion}

Arthroscopy is increasingly used to address hip pathology. A perfect knowledge of the hip joint anatomy and of the surrounding structures is an essential requirement for a secure and successful surgery. Furthermore entry portals for central and peripheral compartment are important: the surgeon should know the areas that may be visualized and treated from each portal. Those concepts are the key to achieve good results and to short the surgical procedure as much as possible. A perfect knowledge of the hip anatomy and a good arthroscopic experience are essential to avoid complication and to optimize the results.

The Authors declare that this article was conduct according ethically to international standards and as required by the journal as described ${ }^{17}$.

\section{References}

1. Robertson WJ, Kelly BT. The safe zone for hip arthroscopy: a cadaveric assessment of central, peripheral, and lateral compartment portal placement. Arthroscopy. 2008;24:1019-1026.

2. Byrd JWT. Hip arthroscopy. J Am Acad Orthop Surg. 2006; 14:433-444.

3. Mella C. Artroscopia de cadera. Santiago, Chile. 2014 Spanish.

4. Byrd JWT, Pappas JN, Pedley MJ. Hip arthroscopy: an anatomic study of portal placement and relationship to the extraarticular structures. Arthroscopy. 1995;11:418-423.

5. Gautier E, Ganz K, Krugel N, Gill T, Ganz R. Anatomy of the medial femoral circumflex artery and its surgical implications. J Bone Joint Surg Br. 2000;82:679-683.

6. Bond JL, Knutson ZA, Ebert A, Guanche CA. The 23-point arthroscopic examination of the hip: basic setup, portal placement, and surgical technique. Arthroscopy. 2009;25(4):416429.

7. Thorey F, Ezechieli M, Ettinger M, Albrecht UV, Budde S. Access to the hip joint from standard arthroscopic portals: a cadaveric study. Arthroscopy. 2013;29(8):1297-307. 
8. Ilizaliturri VM Jr, Byrd JW, Sampson TG, et al. A geographic zone method to describe intra-articular pathology in hip arthroscopy: cadaveric study and preliminary report. Arthroscopy. 2008:24:534-539.

9. Byrd JWT. Gross anatomy. In: Byrd JWT, ed. Operative hip arthroscopy. Ed 2. New York: Springer. 2005;69-83.

10. Keene GS, Villar RN. Arthroscopic anatomy of the hip: an in vivo study. Arthroscopy. 1994;10:392-399.

11. Gray AJR, Villar RN. The ligamentum teres of the hip: an arthroscopic classification of its pathology. Arthroscopy. 1997; 13:575-578.

12. Seldes RM, Tan V, Hunt J, Katz M, Winiarsky R, Fitzgerald $\mathrm{RH}$ Jr. Anatomy, histologic features, and vascularity of the adult acetabular labrum. Clin Orthop Relat Res. 2001;382: 232-240.
13. Ganz R, Parvizi J, Beck M, Leunig M, Notzli H, Siebenrock KA Femoroacetabular impingement: a cause for osteoarthritis of the hip. Clin Orthop Relat Res. 2003;417:112-120.

14. Bedi A, Galano G, Walsh C, Kelly BT. Capsular management during hip arthroscopy: from femoroacetabular impingement to instability. Arthroscopy. 2011;27:1720-1731.

15. Khanduja V, Villar RN. Arthroscopic surgery of the hip: current concepts and recent advances. J Bone Joint Surg Br. 2006 88:1557-1566.

16. Aprato A, Jayasekera N, Bajwa A, Villar RN. Peri-articular diseases of the hip: emerging frontiers in arthroscopic and endoscopic treatments. J Orthop Traumatol. 2014;15(1):1-11.

17. Padulo J, Oliva F, Frizziero A, Maffulli N. Muscles, Ligaments and Tendons Journal. Basic principles and recommendations in clinical and field science research: 2016 Update. MLTJ. 2016;6(1):1-5. 